\title{
Assessment and management of oral health in liver transplant candidates
}

\author{
Paulo Sérgio da SILVA SANTOS ${ }^{1}$, Karin Sá FERNANDES ${ }^{2}$, Marina H. C. GALLOTTINI ${ }^{3}$ \\ 1- PhD, Department of Stomatology, Bauru School of Dentistry, University of São Paulo, Bauru, SP, Brazil. \\ 2- MD, Department of Oral Pathology, Dental School, University of São Paulo, São Paulo, SP, Brazil. \\ 3- PhD, Department of Oral Pathology, Dental School, University of São Paulo, São Paulo, SP, Brazil.
}

Corresponding address: Prof. Dr. Paulo Sérgio da Silva Santos - Alameda Octávio Pinheiro Brisolla, 9-75 -17012-901 - Bauru - SP - Brazil - Phone: 5514 3235-8373 - e-mail: paulosss@fob.usp.br

Received: August 02, 2010 - Modification: June 09, 2011 - Accepted: July 08, 2011

\section{ABSTRACT}

\begin{abstract}
iver transplantation has become a standard treatment for end-stage liver disease and Lthe number of recipients has grown rapidly in the last few years. Dental care during pre-transplant workup is important to reduce potential sources of infection in the druginduced immunosuppression phase of liver transplantation. Objectives: The objectives of this study were to document the prevalence of oral abnormalities in patients on a liver transplant waiting list presenting to an urban dental school clinic, discuss the appropriate dental treatment according their systemic conditions and compare their oral manifestations with those of healthy individuals. Material and Methods: A pilot study was conducted involving 16 end-stage liver disease individuals (study group- SG) attending the Special Care Dentistry Center of the University of São Paulo and 16 control individuals (control group- CG) with no liver diseases, receiving dental care at the Dental School of the University of São Paulo. These individuals were assessed for their dental status (presence of oral disease or abnormalities), coagulation status, and dental treatment indications. Results: The patients from SG exhibited a greater incidence of oral manifestations compared with CG $(p=0.0327)$ and were diagnosed with at least one oral disease or condition that required treatment. Coagulation abnormalities reflecting an increased risk of bleeding were found in $93.75 \%$ of the patients. However, no bleeding complications occurred after dental treatment. Conclusions: The patients with chronic liver diseases evaluated in this study exhibited a higher incidence of oral manifestations compared with the control group and had at least one oral disease or abnormality which required dental treatment prior to liver transplantation. Careful oral examination and evaluation of the patient, including laboratory tests, will ensure correct oral preparation and control of oral disease prior to liver transplantation.
\end{abstract}

Key words: Oral health. Dental care. Liver transplantation.

\section{INTRODUCTION}

There several liver conditions that cause chronic or continuing liver inflammation, and the most common causes of end-stage liver disease are chronic viral hepatitis B and C, alcohol-related liver disease, autoimmune hepatitis, primary sclerosing cholangitis, primary biliary cirrhosis, steatohepatitis, liver disorders inherited or present at birth, and drug-induced liver damage ${ }^{2}$.

In Brazil, since 2006, donor liver allocation has been based on the model for end-stage liver disease
(MELD) scoring system. At present, the MELD has been validated on a broad series of patients with liver diseases of various etiologies and severity ${ }^{1}$. The lab values used in the MELD calculation are serum bilirrubin, International Normalization Ratio (INR) and serum creatinine ${ }^{1}$ (Figure 1 ).

The relationship between the introduction of the MELD allocation system in Brazil and a reduction of donor organ waiting list mortality is controversial. However, the number of recipients of liver transplants has grown dramatically in the last few years and is likely to continue to do so in the future ${ }^{1}$. 


\begin{tabular}{|c|c|}
\hline Laboratory tests & Value labs \\
\hline Bilirrubin & $\mathrm{mg} / \mathrm{dl}$ \\
\hline Creatinine & $\mathrm{mg} / \mathrm{dl}$ \\
\hline INR & $\%$ \\
\hline $\begin{array}{c}3.8[\mathrm{Ln} \text { serum bilirubin }(\mathrm{mg} / \mathrm{dL})]+11.2[\mathrm{Ln} \\
\text { INR] }+9.6[\text { Ln serum creatinine }(\mathrm{mg} / \mathrm{dL})] \\
+6.4\end{array}$ & level \\
\hline
\end{tabular}

Figure 1- Model for end-stage liver disease (MELD) calculation

The improved survival rates and transplant outcomes is predicated upon proper screening and evaluation, as well as breakthroughs in surgical techniques and immunosuppressive therapies. Despite these breakthroughs, infection continues to be a leading cause in graft loss or death of transplant recipients. Although evaluation for chronic systemic infections in transplant recipients is standard, this cannot be said of pre-transplantation dental evaluation ${ }^{2,9}$.

A survey of United States organ transplant centers, conducted between 2003 and 2004, found that among the 294 respondents, 28 ( $9 \%$ ) of organ transplant centers reported one or more incidents of sepsis of dental origin in transplant recipients ${ }^{4}$. In this same survey, $34(11 \%)$ transplant centers reported experiencing one or more episodes of a dental infection prior to transplantation that needed cancellation or postponement of the surgery.

A study performed by the Starzl Transplant Institute involving 300 candidates for liver transplantation conducted between January 2004 and March 2005, reported that oral health attributes (i.e., gingivitis, dental plaque, dental caries, periodontal disease, edentulism, and xerostomia) were similar to those seen in the general population. Candidates for liver transplantation who have not had a dental evaluation for more than 1 year were significantly more likely to present with neglected oral health, untreated dental conditions or habits (e.g., smoking) that were potential precursors and risk factors for dental disease ${ }^{3}$.

Providing oral health care pre- and post-liver transplantation is essential for a better prognosis and quality of life of transplant recipients. The main concern before the transplantation is to eliminate oral foci of infection, such as those of periapical and periodontal origin. During the post-transplant period, the dental professional must be aware of the increased susceptibility to infection in the patient, and the risk for organ rejection, both of which emphasize the importance of vigilant oral health maintenance. Patients are severely immunocompromised and must be well-educated and treated for the rest of their post-transplant lives ${ }^{9}$.

The objectives of this study were to document the prevalence of oral disease and abnormalities in patients on a liver transplant waiting list presenting to an urban dental school clinic, discuss the appropriated dental treatment, according their systemic conditions and compare the oral manifestations with those of healthy individuals.

\section{PATIENTS AND METHODS}

This study was approved by the Research Ethics Committee of the School of Dentistry of the University of São Paulo. All patients signed an informed consent form.

A pilot study was conducted involving 16 endstage liver disease individuals (study group - SG) attending the Special Care Dentistry Center of the University of São Paulo and 16 control individuals (control group - CG) with no liver diseases, who were receiving dental care at the Dental School of the University of São Paulo. SG was formed by all patients with end-stage liver disease that had been referred to the Special Care Dentistry Center for routine dental treatment.

Medical history review and physical examination, with data recorded on a form specifically designed for this study, were compiled in a special form for all the patients.

All patients were questioned about variables such as demographics and history of liver disease. Coagulation studies [i.e., prothrombin time (PT)/ International Normalization Ratio (INR), activated partial thromboplastin time (aPTT), and platelet count] were also performed on all patients.

The dental status of each patient was evaluated at the initial visit by two trained oral medicine dentists. The patient evaluation consisted of a clinical examination of the hard and soft oral tissues and a radiographic examination which consisted in panoramic and periapical radiography. All abnormalities detected in each patient were recorded using a special form designed for this study.

Based upon each patient's dental treatment indications and needs, a dental treatment plan was formulated, and specific dental management recommendations for each patient were presented.

\section{RESULTS}

Among 16 individuals from SG, 13 were male and 3 were female, ranging in age from 37 to 68 (median age of 51 years). Of the 16 individuals from CG, 13 were male and 3 were female, ranging in age from 34 to 70 (median age to 50 years).

The patients with end-stage liver disease presented hepatitis $\mathrm{C}$ and alcohol related liver disease $(5 / 16 ; 31.25 \%)$, hepatitis C $(5 / 16 ; 31.25 \%)$, alcohol-related liver disease $(3 / 16 ; 18.75 \%)$, hepatitis and alcohol related liver disease B (2/16; 
$12.5 \%)$ and Wilson disease (1/16; 6.5\%) (Figure 2). Abnormal coagulation test results, reflecting an increased risk of bleeding, were found in 15 patients from SG $(15 / 16 ; 93.75 \%)$. The most common finding was an abnormally low platelet count (56 to $\left.96 \times 10^{9} / \mathrm{L}\right)$ found in 11 patients $(11 / 16 ; 68.75 \%)$, followed by prolonged PT ( 10 to $52 \mathrm{~s}$ ) in 13 patients $(13 / 16 ; 81.25 \%)$, prolonged aPTT (52 to $46.9 \mathrm{~s})$ in 5 patients $(5 / 16 ; 31.25 \%)$, and increased INR $(>1.3)$ in 7 patients $(7 / 16 ; 43.75 \%)$.

Data collected from oral and radiographic examinations showed that all patients from SG $(100 \%)$ were diagnosed with least one oral disease or condition that would require dental treatment prior to liver transplantation. Caries was diagnosed in 13 patients $(13 / 16 ; 81.25 \%)$; periodontal disease in 11 patients $(11 / 16 ; 68.75 \%)$; petechiae in 3 patients $(3 / 16 ; 18.75 \%)$; oral candidiasis in 2 patients $(2 / 16$; $12.5 \%)$; and gingival overgrowth, ulceration caused by cytomegalovirus (CMV), xerostomia and angular cheilitis were diagnosed 1 patient (1/16;6.25\%). The patients from CG presented caries (7/16; $43.75 \%)$, periodontal disease $(10 / 16 ; 62.5 \%)$ and oral candidiasis $(1 / 16 ; 6.25 \%)$.

The diagnosis of ulceration caused by CMV was detected with PCR real time. Gingival overgrowth was associated with severe periodontal disease and poor oral hygiene. The petechiae were associated in the three cases with thrombocytopenia.

The individuals from SG exhibited a higher incidence of oral diseases compared with those from CG $(p=0.0327)$. Figure 3 summarizes the oral

\begin{tabular}{|c|c|c|c|}
\hline Patients & Age & Subject & Liver diseases diagnoses \\
\hline 1 & 44 & Male & Hepatitis type B and alcohol-related liver disease \\
\hline 2 & 52 & Male & Hepatitis type C \\
\hline 3 & 46 & Male & Hepatitis type C and alcohol-related liver disease \\
\hline 4 & 37 & Male & Hepatitis type C and alcohol-related liver disease \\
\hline 5 & 47 & Male & Hepatitis type C \\
\hline 6 & 43 & Male & Hepatitis type C and alcohol-related liver disease \\
\hline 7 & 54 & Male & Alcohol-related liver disease \\
\hline 8 & 57 & Male & Hepatitis type C and alcohol-related liver disease \\
\hline 9 & 43 & Male & Wilson disease \\
\hline 10 & 60 & Male & Hepatitis type B and alcohol-related liver disease \\
\hline 11 & 44 & Female & Hepatitis type C \\
\hline 12 & 60 & Male & Hepatitis type C \\
\hline 13 & 68 & Female & Alcohol-related liver disease \\
\hline 14 & 43 & Male & Alcohol-related liver disease \\
\hline 15 & 55 & Male & Hepatitis type C and alcohol-related liver disease \\
\hline 16 & 61 & Female & \\
\hline
\end{tabular}

Figure 2- Characteristics of the liver transplant candidates

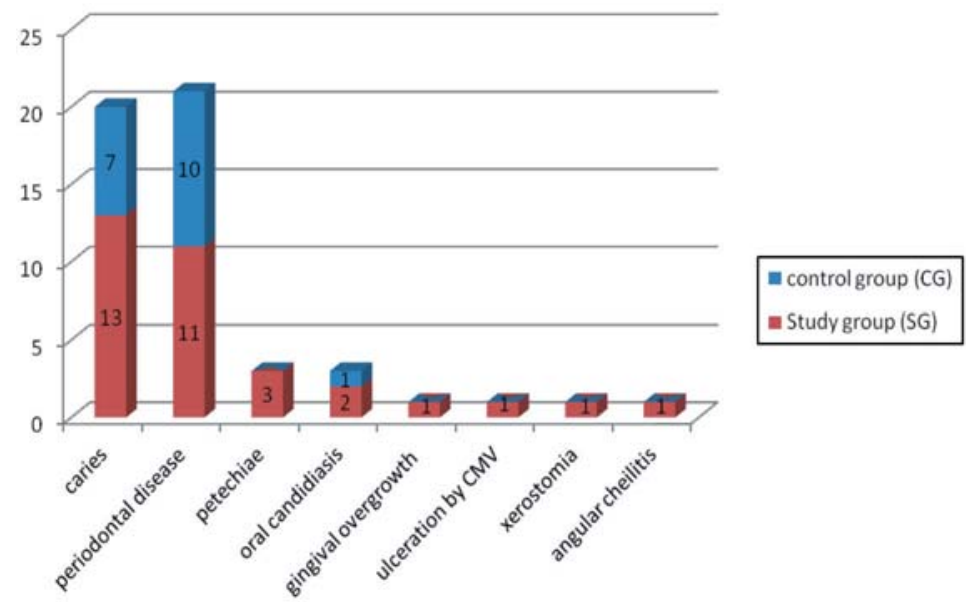

Figure 3- Oral manifestations of study group and control group patients $\mathrm{CMV}=$ cytomegalovirus 
Table 1- Oral manifestations and coagulation abnormalities

\begin{tabular}{|c|c|c|c|c|c|}
\hline \multirow[t]{2}{*}{ Patients } & \multirow[t]{2}{*}{ Oral manifestations } & \multicolumn{4}{|c|}{ Labs values } \\
\hline & & $\begin{array}{c}\text { Platelet } \\
\text { (56 to } 96 \times 109 / L)\end{array}$ & $\begin{array}{c}\text { aPTT } \\
\text { (46.9 to } 52 \mathrm{~s})\end{array}$ & $\begin{array}{c}\text { PT } \\
\text { (10 to } 52 \mathrm{~s})\end{array}$ & $\begin{array}{l}\text { INR } \\
(>1.3)\end{array}$ \\
\hline 1 & $\mathrm{PD}, \mathrm{GO}$, caries & 70 & 49 & 8 & 1.1 \\
\hline 2 & PD, caries & 62 & 39.25 & 15.15 & 1.26 \\
\hline 3 & $\mathrm{PD}$ & 99 & 42 & 10 & 0.72 \\
\hline 4 & PD, caries, OC & 56 & 51 & 7 & 1.6 \\
\hline 5 & DP, caries & 54 & 47 & 15 & 1.15 \\
\hline 6 & caries & 42.6 & 45.7 & 14.5 & 1.82 \\
\hline 7 & DP, caries & 50 & 44.6 & 24.3 & 1.66 \\
\hline 8 & PTC, OC, AC & 96 & 46.3 & 25.7 & 1.54 \\
\hline 9 & $\begin{array}{c}\text { PD, caries, PTC, CMV, } \\
\text { xerostomia }\end{array}$ & 41 & 41.5 & 19.6 & 1.52 \\
\hline 10 & PD, caries, PTC & 64.5 & 46.9 & 18 & 2.67 \\
\hline 11 & caries & 257 & 37 & 13 & 1.14 \\
\hline 12 & PD, caries & 101 & 36 & 14 & 1 \\
\hline 13 & PD, caries & 336 & 25.2 & 14.9 & 0.9 \\
\hline 14 & caries & 290 & 29.5 & 12.9 & 1.12 \\
\hline 15 & caries & 38 & 31.8 & 17.3 & 1.31 \\
\hline 16 & PD, caries & 18 & 35.2 & 16.7 & 1.29 \\
\hline
\end{tabular}

Legend: $\mathrm{PD}=$ periodontal disease; $\mathrm{GO}=$ gingival overgrowth; $\mathrm{OC}=$ oral candidiasis; $\mathrm{PTC}=$ petechiae; $\mathrm{AC}=$ angular cheilitis; $\mathrm{CMV}=$ ulceration caused by cytomegalovirus; aPTT=activated partial thromboplastin time; PT=prothrombin time; INR=international normalization ratio

conditions found in all individuals enrolled in this study. Table 1 shows the oral manifestations from SG and abnormalities coagulation.

After the initial exam and diagnosis of oral diseases, indicated dental treatment procedures were performed on all patients (SG and CG) by the same dentists and included 20 restorations, 21 periodontal scaling and root planning procedures, 6 extractions, 1 incisional biopsy, 3 brush (exfoliative) cytology exams, 2 prosthetic rehabilitations (1 removable prosthesis and fixed prosthesis) and 2 endodontic treatments.

Although the majority of patients showed abnormal coagulation values, none of them exhibited critical values that would represent a contraindication for invasive dental treatment. For this reason, the dental treatments were conducted in our outpatient clinic. Procedures that involve bleeding, such as dental extraction, biopsy and periodontal treatment, were conducted using hemostatic local measurements, such as tranexamic acid paste and absorbable gelatin sterile sponge, as recommended

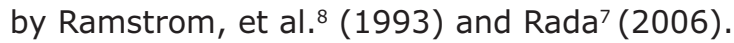

\section{DISCUSSION}

Transplant centers are very specialized facilities that are usually located at university teaching hospitals or large medical centers. They require a large staff of surgeons and other professionals to evaluate and select patients, and perform surgery and follow-up care. However, even though patients are on waiting lists for matching donor livers for time enough to receive definitive dental treatment, this is not a major priority on the protocols of some transplant centers in our city.

The individuals from SG exhibited a higher incidence of oral manifestations compared with CG $(p=0.0327)$ and all of them were diagnosed with at least one oral abnormality that required dental treatment prior liver transplantation in order to avoid potential complications due to infection after transplantation secondary to drug-induced, antiorgan rejection immunosuppression.

The major concern for dental management of patients with end-stage liver disease is the occurrence of bleeding disorders. Routine dental procedures such as dental prophylaxis, tooth extractions, minor periodontal surgeries, and even administration of local anesthetic for restorations can result in serious complications if the dentist is not 
aware of potential risk for impaired hemostasis and increased bleeding in these patients. It is generally accepted that minimally invasive procedures such as simple restorations, supra-gingival prophylaxis, and prosthodontic procedures do not usually involve a considerable risk of bleeding when compared with significantly more invasive procedures such as periodontal surgery and multiple extractions. The difficulty lies is assessing bleeding risk for procedures with a moderate level of invasiveness such as subgingival scaling (especially in the presence of more severe periodontal disease) and extensive (e.g., subgingival) restorations.

In patients with mild to moderate hemostatic impairment, (i.e., INR not greater than 3.5, or a platelet count not less than $50 \times 10^{\circ} \mathrm{L}$ ) careful surgical technique, including an attempt at obtaining primary wound closure and use of local hemostatic measures will usually suffice. The use of absorbable hemostatic materials improves clot formation and stability. However, these materials also carry a risk of infection and may delay healing and should therefore be avoided in immunosuppressed patients. Topical thrombin is an effective agent when applied directly on the bleeding wound as it converts fibrinogen to fibrin and allows rapid hemostasis in a wound. Topical fibrin glue can reduce the amount of factor replacement needed when used along with antifibrinolytic agents. Oxidized cellulose tranexamic acid rinses, astringents (e.g., aluminum chloride), microfibrillar collagen, thrombin-soaked gauze, fibrin sealant and adhesive, electrocautery, absorbable gelatin sponges, and amino-caproic acid (EACA) to prevent clot lysis, have all been suggested as aids to hemostasis in this setting 5,6,10.

Patients with liver failure from any cause are difficult to evaluate from the standpoint of risk for oral bleeding. A relatively small elevation of the PT test (e.g., $\mathrm{PT}=13-15 \mathrm{~s}$ ) suggest significant (i.e., greater than $50 \%$ ) liver damage.

There are various systemic methods that can be used to help compensate for the coagulopathies seen in these patients and decrease the risk of prolonged bleeding from invasive dental procedures. Vitamin K (at doses of $10 \mathrm{mg}$ IM) corrects hypoprothrombinemia from malnutrition and biliary obstruction, but not when due to intrinsic liver disease. Fresh frozen plasma will lower the PT, and platelet transfusion addresses both quantitative and qualitative platelet defects. In the case of more significant coagulopathies, the approach to an invasive procedure may need to be adjusted accordingly, and the patient should be observed postoperatively in the surgery for a longer time period than usual ${ }^{5,6}$.

Care should be exercised with the use of narcotic analgesics (e.g., morphine) and sedatives (e.g., diazepam), as their duration of action may be prolonged in patients with decompensated liver disease. As with chronic renal failure, reduced doses should be used for drugs that are metabolized by the liver. In particular, avoid the use of paracetamol in the presence of liver failure and alcoholism, although doses of up to $4 \mathrm{~g}$ /day for up to 2 weeks are allowable. Patients should be strongly advised not to take alcohol during the period when they are using acetaminophen.

Careful oral examination and evaluation of the patient, including laboratory tests, will ensure correct oral preparation and control of oral disease prior to liver transplantation. Patients with chronic liver diseases may present at any dental office for treatment and therefore general dentists should be aware of the unique concerns involving their assessment, education, treatment and maintenance of oral health.

\section{CONCLUSION}

The patients with chronic liver diseases evaluated in this study exhibited a higher incidence of oral manifestations compared with the healthy control group and had at least one oral disease or abnormality that required dental treatment prior to liver transplantation.

\section{REFERENCES}

1- Dente K. Brazil developing solutions for handling limited liver transplantation resources [online]. Medscape Today. s. d. [cited August 19th 2011]. Available from: <http://www.medscape.com/ viewarticle/558856>.

2- Douglas LR, Douglass JB, Sieck JO, Smith PJ. Oral management of the patient with end-stage liver disease and the liver transplant patient. Oral Surg Oral Med Oral Pathol Oral Radiol Endod. 1998;86:55-64.

3- Guggenheimer J, Eghtesad B, Close JM, Shay C, Fung JJ. Dental health status of liver transplant candidates. Liver Transpl. 2007;13:280-6.

4- Guggenheimer J, Mayher D, Eghtesad B. A survey of dental care protocols among US organ transplant centers. Clin Transplant. 2005; 19:15-8.

5- Gupta A, Epstein JB, Cabay RJ. Bleeding disorders of importance in dental care and related patient management. J Can Dent Assoc. 2007;73:73-83.

6- Lockhart PB, Gibson J, Pond SH, Leitch J. Dental management considerations for the patient with an acquired coagulopathy. Part 1: Coagulopathies from systemic disease. Br Dental J. 2003;195:439-45.

7- Rada RE. Management of the dental patient on anticoagulant medication. Dent Today. 2006;25:58-63.

8- Ramström G, Sindet-Pedersen S, Hall G, Blombäck M, Alander $U$. Prevention of postsurgical bleeding in oral surgery using tranexamic acid without dose modification of oral anticoagulants. J Oral Maxillofac Surg. 1993;51:1211-6.

9- Wakefield CW, Throndson RR, Brock T. Liver transplantation: dentistry is an essential part of the team. J Tenn Dent Assoc. 1998;75:9-16.

10- Ward BB, Weideman EM. Long-term postoperative bleeding after dentoalveolar surgery in the pretransplant liver failure patient. J Oral Maxillofac Surg. 2006;64:1469-74. 